\title{
Analyse De L'unité Morphologique Comme Signe Linguistique À Double Face Destinée Aux Apprenants Universitaires Au Nigéria : Le Cas De La Flexion Verbale En Français
}

\author{
Mabel Ejoghoye Aziegbe \\ Efeovbokhan Goodnames Gooddyy Ogbevuoen \\ Department of Modern Languages, Ambrose Alli University, \\ Ekpoma, Edo State, Nigeria
}

\section{Résumé}

Cet article porte sur l'analyse de la flexion verbale en français. En tant que professeurs de français et chercheurs en linguistique appliquée à l'enseignement du français langue étrangère, nous avons constaté que l'analyse morphématique (ou l'analyse en morphèmes), un aspect de la morphologie, semble être négligée dans le domaine de l'analyse des éléments de la langue française par les auteurs des ouvrages consacrés à la grammaire française et par les enseignants de la grammaire française dans les universités nigérianes, nous considérons la prise en compte de ce sujet comme très important. L'étude a comme but de produire un matériel pédagogique dans le domaine en question et d'attirer l'attention des autres chercheurs à cet aspect de l'analyse des éléments du langage qui semble être abandonné. Avant d'aborder le sujet proprement dit, il nous est nécessaire de montrer l'investigation que nous avons faite dans le but de confirmer que l'analyse en morphèmes subit cet abandon. Ainsi pour cette étude, nous nous somme servis de deux groupes de données: les données recueillies grâce à la compilation de la liste de dix (10) ouvrages pédagogiques de grammaire française dans lesquels plusieurs sortes d'analyse des éléments de la langue francise (l'analyse grammaticale, l'analyse du groupe fonctionnel et l'analyse logique) sont traitées et les données obtenues par le questionnaire administré à quinze (15) professeurs enseignant la grammaire française au Village français du Nigeria (le Centre interuniversitaire nigérian d'études françaises) / «Nigeria French Language (Inter-University centre for French Studies), Badagry, Lagos State » et dans sept (7) universités nigérianes. Après l'analyse des données recueilles, le résultat suggère que l'analyse en morphèmes semble être négligée.Ensuite, nous 
avons fait une analyse en morphèmes de la flexion verbale en français pour servir d'exemple de l'analyse en morphèmes. Cet article est considéré comme contribution à la production de documents pédagogiques destinés à l'enseignement et à l'apprentissage de l'analyse morphématique en français chez les apprenants du français langue étrangère, surtout ceux dans les universités nigérianes.

Mots clés: analyse morphématique, flexion verbale, unité morphologique, signifiant, signifié

\title{
Analysis of Morphological Unit as Double Face Linguistic Sign for theUniversity Students in Nigeria: The Case of Verb InflectionalMorphemes inFrench
}

\author{
Mabel Ejoghoye Aziegbe \\ Efeovbokhan Goodnames Gooddyy Ogbevuoen \\ Department of Modern Languages, Ambrose Alli University, \\ Ekpoma, Edo State, Nigeria
}

Abstract

This article focuses on analysis of verb inflectional morphemes in French. As teachers of French and researchers in linguistic applied to the teaching of French as a foreign language, we have noticed that morphematic analysis in French seems to be neglected in the domain of analysis of language elements by authors of books on French grammar and teachers of French grammar in Nigerian universities, we consider the choice of this topic as very important. The objectives of the study are to produce a pedagogical material in the domain in question and to draw the attention of other researchers to this aspect of analysis of linguistic elements in French which seems to be neglected. Before treating the main subject of our study, we have deemed it wise to present the investigation which we carried out to confirm that morphematic analysis in French seems to be neglected. Hence for this study, we made use of two sets of data: the data realized through the compilation of the list of ten (10) books on French grammar where different types of analysis of linguistic elements (grammatical analysis at the levels of words, groups and clauses) in French are treated and the data collected from the questionnaire administered to 15 teachers of French grammar in Nigeria French Language (Inter-University centre for French Studies ) and in 
seven (7) Nigerian universities. The result of the study suggests that morphematicanalysis in French seems to be neglected by authors of books on French grammar and teachers of French grammar in Nigerian universities.Afterwards, we did an analysis of verb inflectional morphemes in French, to serve as an example of morphematic analysis. This article is regarded as contribution to the production of teaching materials towards the promotion of the teaching and learning of morphematic analysis in French among foreign learners of French, especially those in Nigeria.

Keywords: Morphematic analysis, verb inflectional morpheme, morphological unit, signifier, signified

\section{Introduction}

Cette étude se situe au domaine de la morphologie, une branche de la linguistique qui étudie le système des morphèmes. L'étude morphologique en français comprend deux aspects: l'une qui concerne la morphologie flexionnelle (la conjugaison : être = je suis, tu es... ), la formation du pluriel des substantifs : livre $+\mathrm{s}=$ livres), la morphologie dérivationnelle (la formation de l'adverbe : belle + ment $=$ bellement) et la composition( nom compose : bon + homme = bonhomme), et l'autre qui se consacre à l'analyse morphématique (ou en l'analyse morphèmes). Celle-ci nous intéresse dans cet article. Le terme analyse morphématique s'emploie chez Touratier (2002) pour désigner l'aspect de la morphologie dont la spécificité est l'identification proprement dite des morphèmes, laquelle repose et sur des considérations morphologiques et sur des considérations sémantiques, dans la mesure où les morphèmes sont des unités à deux faces, une face signifiant et une face signifié. L' 'analyse en morphèmes fait partie de l'analyse des éléments du langage, qui peut se faire, en grammaire structurale, à toutes les différentes unités linguistiques. L'unité linguistique est un élément discret identifié à un certain niveau (ou rang). Ainsi, les morphèmes sont des unités grammaticales (au rang morphématique), les mots (au rang du mot), les groupes (au rang du groupe) et les phrases (au rang phrastique). Chaque unité linguistique est définie par les rapports qu'elle entretient avec les autres unités linguistiques dans un système donné(Dubois et al., 2002). C'est pourquoi, par exemple, chacun des quatre types d'analyse présentés ci- dessous, correspond à une unité linguistique:

- L'analyse en morphèmes prend pour unité le morphème: $\mathrm{il} / \mathrm{s}$ / march/ent / lent/ el ment

- L'analyse grammaticale prend pour unité le mot: ils, marchent, lentement

- L'analyse par groupe fonctionnel prend pour unité le groupe de 
mots: les garçons, marchent lentement

- L'analyse logique prend pour unité la proposition: Les étudiants

marchent lentement;

ils sont en retards.

On trouve que maints ouvrages pédagogiques de grammaire française contiennent un chapitre ou une section où l'on trouve soit l'analyse grammaticale, soit l'analyse du groupe fonctionnel, soit enfin l'analyse logique (voir la partie méthodologique de ce travail). Mais à notre connaissance, on n'y trouve rien sur l'analyse en morphèmes. Néanmoins, il existe, dans ce domaine, l'ouvrage fondamental de Touratier (2002) intitulé Morphologie et morphématique: Analyse en morphèmesoù l'auteur luimême affirmeque, en français, il manque de manuel d'analyse morphématique. Voici ses propres mots: "Curieusement, alors que le morphème est une des grandes innovations théoriques de la linguistique moderne, il n'y a pas eu en français de manuel d'analyse en morphèmes. A la belle époque de la linguistique structuraliste,... Nous voudrions donc combler cette lacune".

Nous considérons la prise en compte de ce sujet comme très important, puisque l'analyse des éléments du langage (exemple: l'analyse grammaticale et l'analyse logique) ainsi que l'étude morphologique fait partie du programme de français dans les universités nigérianes. Donc cette étude a pour objet de produire un ouvrage pédagogique dans le domaine en questions et d'attirer l'attention des autres chercheurs à cet aspect de l'analyse des éléments du langage qui semble être négligée.

\section{Quelques notions en linguistique}

Il convient de présenter quelques notions en linguistique afin de bien comprendre cet article. Cela est dû au fait que rare sont les grammaires d'une langue particulière qui les utilisent vraiment (Touratier, 2002).

\section{1. Le morphème}

Les morphèmes sont les éléments grammaticaux abstraits, qui sont réalisés par les morphes. En grammaire linguistique structurale, le morphème est le constituant immédiat du mot (Dubois et al., 2002 ). Il est la plus petite unité linguistique ayant une forme et un sens (Moeschler \& Auchlin, 2005 ). Il est le constituant immédiat isolable comme segment dans l'énoncé. On isole les morphèmes par segmentation. Par exemple, il y a neuf (9) morphèmes dans l'énoncé : leur/s livre/s ét/ ai/ent ouvert/s. En outre, le mot chanteurs comporte trois (3) morphèmes (chant/eur/s) qui sont liés. Au contraire, on ne peut diviser les mots vapeur, moustique en les éléments distincts (vapeur $=* v a /$ peur $;$ moustique $=*$ mou/ stique). Chacun de ces deux mots constitue donc un seul morphème. 


\section{Les différents types de morphèmes}

Selon Rohr (s.d.), les morphèmes peuvent se répartir en trois groupes suivants:

\section{a. Les morphèmes liés et les morphèmes libres}

Les morphèmes liés sont toujours en combinaison avec d'autres morphèmes, c'est-à-dire ils ne peuvent pas apparaître isolés:

$\boldsymbol{r e}$-dans refaire ; im-dans impossible;

$-\boldsymbol{s}$ dans les, grands, manges

Les morphèmes libres (ou non liés) se rencontrent sous forme libre ou non liée:

-faire dans refaire; -possible dans impossible; -face dans préface; le-dans les; grand- dans grands; mange-dans manges ; leur-dans leurs ; il-/ elle- dans ils/ elles ; belle- dans bellement

\section{b. La base et l'affixe}

La base (ou radical) du mot lui donne son identité sémantique. À cette base on peut ajouter des affixes qui ont pour fonction d'ajouter une signification ou de modifier le sens de la base

Exemples:

garçon- dans garçons ; arriv- dans arriver

\section{c. Les morphèmes lexicaux etles morphèmes grammaticaux}

Les morphèmes lexicaux sont les radicaux, ou bases, les mots simples, porteurs du sens de base; auxquels s'ajoutent les affixes (préfixes, suffixes) ; ils sont en une classe ouverte, avec des créations ((par emprunt ou formation propre) et des disparitions. Ce sont les morphèmes qui correspondent aux mots du dictionnaire, qui disposent d'une certaine autonomie (Moeschler \& Auchlin, 2005).

Exemples:

fin- dans finir ; active- dans activement

grand-dans grands; chant- dans chanteur

Les morphèmes grammaticaux (affixes ou mots grammaticaux). Ils constituent un ensemble clos et limite, en raison de leur contenu, qui est l'expression d'un petit nombre de catégories et relation (Moeschler \& Auchlin, 2005). Les affixes sont des morphèmes grammaticaux non autonomes (nécessairement associés à une racine).

Exemples:

$-\boldsymbol{s}$ dans garçons et grands; - $\boldsymbol{e r}$ dans arriver -ir dans finir ; -ment dans activement ; -eur dans chanteur 


\section{Types d'affixes(ou morphèmes liés)}

En français, on peut distinguer les affixes selon la place qu'ils occupent par rapport au radical: préfixe, suffixe.Les affixes qui sont placés avant le radical s'appellent les préfixes (re- dans le mot refaire); ceux qui se placent après le radical sont les suffixes (-euse, -ment dans les mots chanteuse, heureusement, activement). Le mot incroyablement contiennent le préfixe in- et les suffixes -able, -ment).

Les affixes peuvent aussi se classer d'après la fonction: les affixes dérivationnels et les affixes flexionnels. Les affixes dérivationnels servent à former avec un radical un thème capable de fonctionner comme verbe, nom, adjectif ou adverbe. Ils expriment des contenus quasi lexicaux. Par exemples, des mots construits grâceaux affixes dérivationnels sont présentes ci-dessous:

i. Exemples des mots formés à l'aide de préfixes:

des noms: préface, postface, coéquipier, antivol...

des verbes: relire, refaire,

des adjectifs: impossible, inactif...

ii. Exemples des mots formés à l'aide de suffixes:

a. Des noms formés:

- à partir d'un verbe : augmente = l'augmentation ;

montent $=$ la montée $;$ chante $=$

le chanteur ; sécher = le séchoir, etc.

- à partir d'un nom: une assiette = une assiettée ;

une mangue = un manguier ;

un garçon = un garçonnet ;

une fille $=$ fillette

- à partir d'un adjectif : douce = la douceur ; dure = la duretél durée $;$ beau = beauté

b. Des verbes sont formés à partir d'un adjectif: grand $=$ grandir; maigre $=$ maigrir $;$ noir $=$ noircir; égal = égaler

c. Des adjectifs sont formés :

- à partir d'un nom : université = universitaire

- à partir d'un verbe : manger = mangeable

d. Des adverbes sont formés à partir d'un adjectif qualificatif: active = activement $;$ admirable $=$ admirablement ; belle $=$ bellement $;$ certaine $=$ certainement ; égale $=$ également $;$ gaie $=$ gaiement ;

hardi $=$ hardiment $;$ lent $=$ lentement ; parfaite $=$ parfaitement $;$ petite $=$ petitement ; poli $=$ poliment $;$ telle $=$ tellement $;$ vive $=$ vivement 


\section{Les affixes flexionnels:}

Lesaffixes flexionnels expriment des catégories grammaticales en nombre limité: nombre, genre, personne, cas, temps verbal, etc. (Moeschler \& Auchlin, 2005). En français, ils regroupent les suivants: les flexions nominal et adjectival, et lesdésinences verbales. Les flexions nominales et adjectivales apportent des informations grammaticales sur les noms et les adjectifs:

- le $-s$ du pluriel est une flexion nominale ou adjectivale: garçons,petits

- le - $e$ du féminin est une flexion nominale ou adjectivale: une avocate, une amie une voiture noire; une grande salle

Pour les verbes, la flexion (conjugaison) détermine:

- la personne: La personne sert à exprimer la relation entre le verbe et son sujet $\left(1^{\mathrm{er}}, 2^{\mathrm{e}}, 3^{\mathrm{e}}\right)$

- le nombre : Le nombre affecte les 3 personnes qui peuvent avoir un singulier (je, tu, il/ elle) et un pluriel (nous, vous, ils/elles).

- le mode: Le mode exprime l'idée verbale selon son degré d'actualisation. En français il y a 7 modes: l'indicatif, le subjonctif, le conditionnel, l'impératif (les modes personnels) et le participe présent et passé, le gérondif et l'infinitif (les modes non-personnels ou impersonnels).

- le temps : Le temps sert à situer l'action ou l'état exprimé par le verbe dans la chronologie. Ceci vaut pour l'indicatif, valeur temporelle, dans les autres cas on parlera de valeur aspectuelle (Rohr, s.d.).

\section{2. Le morphe, l'allomorphe et le morphe portemanteau}

Le morphe est le constituant immédiat isolable comme segment dans l'énoncé, et représentant du ou des morphème(s) (Dubois et al., 2002 ). Par exemple, le mot dansons est composé de:

- le morphe /dans/ correspondant au morphème lexical \{danser\}

- le morphe /ons/ correspondant aux morphèmes grammaticaux $\{$ présent $\}+\{$ pluriel $\}+\left\{2^{\mathrm{e}}\right.$ personne $\}$.

On appelle allomorphes les diverses représentations segmentales du morphème unique. Autrement dit, un morphème unique peut être réalisé par plusieurs morphes (allomorphes). En effet, un allomorphe est défini comme une variante d'un morphème que l'on trouve dans certains contextes définis (Gleason, 1967 cité par Rohr, s.d.). Ainsi, le morphème lexical \{aller\} est-il réalisé en français par les morphes (allomorphes) /v/, /all/, /i/, /ail/ respectivement, comme le montrent les exemples qui suivent:

/v/: je vais; tu va, il/elle va, ils/elles vont

/all/: nous allons, vous allez, j'allais, tu allais, il/elle allait/...

/i/: j'irai, tu iras, il/elle ira, nous irons...

/aill/: que j'aille, que tu ailles, qu'il /elle aille, qu'ils/elles aillent 
En outre, dansla phrase: leurs livres étaient ouverts, le morphème \{pluriel\} est représenté par deux allomorphes (/ent/ et / s/). Le morphème \{manière\} se réalise par les allomorphes:/ment/, lémen/, lamment/, lemment/ respectivement dans les adverbes:bellement, précisément; suffisamment, évidemment.

Un morphe portemanteau est une unité morphologique qui exprime simultanément plusieurs morphèmes (Moeschler \& Auchlin, 2005). On ne peut pas les segmenter. Ce sont les cas, par exemple, des formes contractées (morphes) résultant de la préposition $\grave{a}$ ou $\boldsymbol{d} \boldsymbol{e}$ et les articles définis le et les $(\grave{a}+l e=a u ; \grave{a}+l e s=a u x ; d e+l e=d u ; d e+l e s$ $=$ des dans les énoncés analysés ci-dessous:

a. Je vais au marché.

- Le morphe /au/ représente simultanément la préposition \{à\} et le déterminant défini masculin, singulier $\{$ le $\}$.

b. Elles parlent aux enfants.

- Le morphe /aux/ exprime simultanément la préposition \{à\} et le déterminant défini masculin, pluriel $\{$ les $\}$.

c. C'est la porte du couloir.

C'est le livre du professeur.

- Le morphe /du/ exprime simultanément la préposition $\{$ de $\}$ et le déterminant défini masculin, singulier $\{l e\}$.

d. C'est le travail des hommes.

- Le morphe $/ d e /$ exprime simultanément la préposition $\{$ de $\}=$ (morphème) et le déterminant défini masculin, pluriel \{les\}.

$C$ 'est le travail des femmes.

- Le morphe /del exprime simultanément la préposition $\{$ de $\}$ et le déterminant défini féminin, pluriel $\{$ les $\}$.

\section{3. L'unité morphologique, signe: l'association signifiant/ signifié}

L'unité de la morphologie est un signe linguistique. Le signe (linguistique) est constitué par l'association d'un signifiant,forme matérielle qui évoque un contenu sémantique et d'un signifié, contenu sémantique évoqué par une forme matérielle (Riegel et al., 2014). L'unité morphologique, comme tout signe linguistique, comporte un signifié, qui est son sens ou sa valeur, et un signifiant grâce à quoi le signe se manifeste (Martine, 1970). En morphologie, les termes morphe et morphème correspondent respectivement aux termes signifiant et signifié.

\section{Méthode de travail}

\section{1. Constitution du corpus/ composition de l'instrument}

Signalons d'abord que notre corpus est de deux types : les données recueillies grâce à la compilation de la liste de quelques ouvrages 
pédagogiques de grammaire française dans lesquels plusieurs sortes d'analyse des éléments de la langue francise sont traitées (voir la partie de l'analyse des résultats du travail) et celles obtenues au moyen du questionnaire. Tandis que les données recueillies grâce à la compilation de la liste de quelques ouvrages pédagogiques de grammaire française a pour but de montrer si les auteurs traitent l'analyse en morphèmes en français, le questionnaire vise à savoir si l'analyse en morphèmes est enseignée ou/et appréciée par les enseignants de la grammaire française dans les universités nigérianes. Pour ce faire, nous avons posé une question visée à reconnaître leur opinion sur l'analyse en grammaire.

\section{2. Sujets testés et lieux d'enquêtes}

La population visée est les professeurs enseignant la grammaire française chez les étudiants universitaires au Nigeria. Pendant l'année académique 2017/2018, un questionnaire a été administré à quinze (15) professeurs enseignant la grammaire française au «Nigeria French Language (Inter-University centre for French Studies ), Badagry, Lagos State » et dans sept (7) universités nigérianes: «Ambrose Alli University, Ekpoma »; «Delta State University, Abraka »; "Lagos State University, Lagos »; " Osun State University, Osogbo »; "University of Benin, Benin »; « University of Ibadan, Ibadan » et University of Ilorin, Ilorin. Ces enseignants constituent un échantillon représentatif des autres enseignants de la grammaire française dans les universitaires nigérianes.

\section{3. Présentation et analyse des résultats}

a. Les données constituées à partir de la compilation de la liste de quelques ouvrages pédagogiques de grammaire française

Nous avons ci-dessous listée dix (10) ouvrages pédagogiques de grammaire française dans lesquels les auteurs touchent à différents types d'analyse des éléments de la langue francise.

Tableau 1

\begin{tabular}{|l|c|c|c|}
\hline \multicolumn{2}{|c|}{ Ouvrages pédagogiques de grammaire française } & \multicolumn{2}{c|}{ Types d'analyses } \\
\hline $\begin{array}{l}\text { 1. Bako (2010) La Grammaire fondamentale du français à } \\
\text { l'usage des parents, des enseignants, des élèves et étudiants } \\
\text { africains }\end{array}$ & $\begin{array}{c}\text { l'analyse } \\
\text { logique }\end{array}$ & $\begin{array}{c}\text { l'analyse } \\
\text { grammaticale }\end{array}$ \\
\hline $\begin{array}{l}\text { 2. Bentolila (2001) Grammaire: La référence pour tous } \\
\text { avec les réponses immédiates à 700 questions d'usage }\end{array}$ & $\begin{array}{c}\text { l'analyse } \\
\text { logique }\end{array}$ & $\begin{array}{c}\text { l'analyse du groupe } \\
\text { fonctionnel }\end{array}$ & $\begin{array}{c}\text { l'analyse } \\
\text { grammaticale }\end{array}$ \\
\hline $\begin{array}{l}\text { 3. Bescherelle (2006) La grammaire pour tous: toutes les } \\
\text { règles, des exemples commentés, des outils d'analyse, un } \\
\text { index des difficultés }\end{array}$ & & $\begin{array}{c}\text { l'analyse } \\
\text { grammaticale }\end{array}$ \\
\hline $\begin{array}{l}\text { 4. Dubois et al. (2002) Dictionnaire de linguistique } \\
\text { l'analyse } \\
\text { logique }\end{array}$ & & $\begin{array}{c}\text { l'analyse } \\
\text { grammaticale }\end{array}$ \\
\hline
\end{tabular}




\begin{tabular}{|c|c|c|c|}
\hline $\begin{array}{l}\text { 5. Dubois \& Lagane (2004) La nouvelle grammaire du } \\
\text { français }\end{array}$ & & l'analyse du groupe & \\
\hline 6. Ezeani (2002) Éléments de Langue française & $\begin{array}{l}\text { l'analyse } \\
\text { logique }\end{array}$ & & \\
\hline 7. Gaillard \& Colignon (2005) Toute la grammaire & & & $\begin{array}{c}\text { l'analyse } \\
\text { grammaticale }\end{array}$ \\
\hline 8. IPAM (2002) Grammaire du français & $\begin{array}{l}\text { l'analyse } \\
\text { logique }\end{array}$ & & \\
\hline $\begin{array}{l}\text { 9. Nekpo (2013) Comment parlons-nous français? Etude } \\
\text { syntaxique commentée de quelques mots et expressions } \\
\text { courants }\end{array}$ & $\begin{array}{l}\text { l'analyse } \\
\text { logique }\end{array}$ & & $\begin{array}{l}\text { l'analyse } \\
\text { grammaticale }\end{array}$ \\
\hline $\begin{array}{l}\begin{array}{l}\text { 10. Sanni (2010) } \\
\text { grammaticale". }\end{array} \\
\text { gram }\end{array}$ & & & $\begin{array}{c}\text { l'analyse } \\
\text { grammaticale }\end{array}$ \\
\hline
\end{tabular}

Tableau II : Analyse du tableau 1

\begin{tabular}{|l|lc|}
\hline \multicolumn{1}{|c|}{ Domaine d'analyse traitée } & \multicolumn{2}{|c|}{ Nombre d'auteurs (\%) } \\
\hline L'analyse logique & $6 / 10$ & $(60 \%)$ \\
\hline L'analyse du groupe fonctionnel & $2 / 10$ & $(20 \%)$ \\
\hline L'analyse grammaticale & $7 / 10$ & $(70 \%)$ \\
& & \\
\hline
\end{tabular}

Le tableau (II) montre que (60\%) des 10 auteurs travaillent sur l'analyse logique. (20\%) d'eux et $(70 \%)$ d'eux traitent respectivement l'analyse du groupe fonctionnel et l'analyse grammaticale dans leurs ouvrages pédagogiques de grammaire française.

Nous constatons, à la lecture du tableau, que l'analyse en morphèmes n'y se figure pas. Donc on peut dire que, d'une manière générale, les auteurs d' ouvrages pédagogiques de grammaire accordent une place prédominante à l'analyse grammaticale, à l'analyse du groupe fonctionnel, enfin à l'analyse logique mais négligent l'analyse en morphèmes.

\section{b. Le questionnaire}

Question: Dans les universités nigérianes, l'enseignement/apprentissage de l'analyse en morphèmes en français devrait être encouragé. Nous souhaitons connaître votre position.

\section{Analyse:}

Pour traiter les informations fournies par nos sujetsen ce qui concerne l'opinion sur l'enseignement de l'analyse en morphèmes aux apprenants de français dans les universités nigérianes, nous avons, d'abord, relevés quelques opinions de leurs productions. Nous les avons classées en deux: les opinions positives (encourageantes) et les opinions négatives (décourageantes) comme le montre le tableau III présenté ci-dessous:

\section{Le tableau III}

\begin{tabular}{|c|c|c|c|}
\hline \multicolumn{2}{|c|}{ Opinion positive (encourageante) } & \multicolumn{2}{|c|}{ Opinion négative ( décourageante) } \\
\hline Nombre & Pourcentage & Nombre & Pourcentage \\
\hline $2 / 15$ & $13,3 \%$ & $13 / 15$ & $86,7 \%$ \\
\hline
\end{tabular}


Ce tableau montrent que(13,3\%) des sujets contactés ont les opinions positives( encourageantes) sur l'enseignement de l'analyse en morphèmes aux apprenants de français dans les universités nigérianes alors que $(86,7 \%)$ en ont les opinions négatives(décourageantes). Les exemples cités dans le tableau IV en témoigne.

Nous présentons, dans le tableau ci-dessous, des opinions positives (encourageantes) et des opinions négatives (décourageantes) fournies par nos sujets.

Le tableau IV

\begin{tabular}{|c|c|}
\hline Opinion positive (encourageante) & Opinion négative (décourageante) \\
\hline $\begin{array}{l}\text { - « Nos étudiants doivent apprendre l'analyse en } \\
\text { morphèmes afin d'avoir des connaissances } \\
\text { approfondies de la morphologie } \\
\text { - «L'enseignement de l'analyse en morphèmes est } \\
\text { nécessaire, mais nos étudiants ont besoin d'abord } \\
\text { de la grammaire traditionnelle » } \\
\text { - Il leur faut l'analyse en morphèmes. Aucune } \\
\text { connaissance n'est perdue. » }\end{array}$ & $\begin{array}{l}\text { - «L'analyse en morphèmes, cet aspect de la } \\
\text { morphologie est destinée aux étudiants de sciences } \\
\text { du langage et non à nos étudiants à qui le } \\
\text { français est une langue étrangère » } \\
\text { - « Comment enseigner l'analyse en morphèmes } \\
\text { chez nos étudiants qui n'arrivent pas à conjuguer les } \\
\text { verbes au passée composé ? (exemple : * je suis } \\
\text { aller et même j'ai aller) » - «L'analyse en } \\
\text { morphèmes sera inutile à nos étudiants. Il leur faut } \\
\text { la grammaire traditionnelle ». } \\
\text { - «L'acquisition de l'analyse en morphèmes n'aide } \\
\text { pas les étudiants à bien s'exprimer. » } \\
\text { - «L'analyse en morphèmes ne joue aucun rôle à la } \\
\text { réalisation des objectifs fixés de la didactique du } \\
\text { français langue étrangère (FLE) (exemple: } \\
\text { formation des étudiants qui peuvent bien travailler } \\
\text { comme secrétaires bilingues, interprètes, etc. » }\end{array}$ \\
\hline
\end{tabular}

De ce qui précède on peut dire que l'analyse en morphèmes semble être négligée par les enseignants de la grammaire française dans les universités nigérianes

\section{L'analyse morphématique: les flexions verbales}

Dans cette partie, qui est le cœur de notre étude, nous analysons des extraits du manuel de Riegel, Pellat, \& Rioul (2014 : 440-441) où ces auteurs présentent des désinences verbales en employant les termes traditionnels: marques temporelles, marques de personne et de nombre,etc.Nous employons les termes morphe et morphème pour designer chacun de ces termes afin de montrer les deux faces que comporte tout signe linguistique. Le morphe est représenté entre barres obliques / / et le morphème, entre accolades \{ \} comme le font Moeschler \& Auchlin (2005).

Cette partie se compose de deux sections. La première section, intitulée le mode impersonnel, présente des extraits basés sur l'infinitif et le participe ainsi que leurs analyses (voir nos. i, ii et iii). La deuxième est 
intitulée le mode personnel; elle comporte des extraits basés sur les marques de temps, de personne et de nombre et leurs analyses respectives (voir nos. iv, v, vi, vii, viii, ix et x).

\subsection{Première Section: Les modes impersonnels (ou non-personnels)}

\section{Extrait i :}

L'infinitif présent comporte à l'écrit quatre désinences possédant un-r commun: -er (chanter), -ir (venir),-oir (voir), -re (battre).

\section{Analyse:}

Les morphèmes grammaticaux \{infinitif\} + \{présent $\}$ se réalisent par plusieurs morphes. Par exemple, il peut se réaliser différemment, selon le verbe type:

- le morphe / er/ : manger danser...

- le morphe / ir/ : couvrir, finir, grandir, venir ...

- le morphe /oir/ : voir, vouloir, pouvoir...

- le morphe / re/ : écrire, faire,prendre rire,...

\section{Extrait ii:}

Le participe présent et le gérondif se forment avec la désinence-ant, généralement sur la base de la première personne du pluriel de l'indicatif (analogue au radical de l'imparfait): finiss-ant (nous finissons, il finissait). Trois verbes font exception: être (étant), ainsi qu'avoir et savoir, dont le participe est formé sur la base du subjonctif présent (ay-ant; sach-ant)

\section{Analyse:}

Le morphe /ant/ correspond aux morphèmes grammaticaux \{participe $\}+\{$ présent $\}$ et au morphème grammatical \{gérondif\}.

Exemples:

Participe présent: marchant, finissant, étant, etc.

Gérondif: (Il parle en) marchant.

\section{Extrait iii:}

Les participes passés: ils sont formés pour la plupart à l'aide d'une voyelle dont le choix dépend de la conjugaison du verbe. Les verbes en -er forme leur participe passé à l'aide de -é (chanté). Les verbes en -ir (finir) et en -ire (dire) ont leurs participes en -i, comme fini, cueilli, sorti; la voyelle peut être suivie d'une consonne - $t$ (écrit) ou -s (acquis). Certains verbes en ir ou -ire forment leur participe passé avec la voyelle -u: : couru, lu, tenu venu, vêtu. Les verbes en -oir et en -re forment leur participe passé avec la voyelle -u: dî, vu, su conclu, rendu, battu, vécu, bu, cru. Certains verbes en -oir et -re prennent la voyelle -i: assis, pris, mis, suivi. 


\section{Analyse:}

Les morphèmes grammaticaux \{participe $\}+\{$ passé $\}$ sont représentés par plusieurs morphes qui varient selon le type de verbe comme le montrent les exemples présentés ci-dessous:

- Le morphe /é/: chanté (chanter)

- Le morphe /i/: fini (finir), cueilli (cueillir), sorti (sortir)

- Le morphe /it/: écrit (écrire), dit (dire)

- Le morphe /u/: couru (courir), tenu (tenir) venu (venir), vêtu(vêtir) lu (lire), du (devoir), vu (voir), su (savoir), conclu (conclure), rendu, (rendre), battu (battre),vécu (vivre), bu (boire), cru (croire)

- Le morphe /is/: assis (asseoir), pris (prendre), mis (mettre)

\section{2. Deuxième Section : Les modes personnels}

\section{2. 1. Les morphes exprimant le temps (ou marques temporelles)}

\section{Extrait iv:}

Plusieurs temps sont marqués, pour tous les verbes, par des éléments qui s'ajoutent immédiatement à radical et sont suivis des marques de personne et de nombre (440):

\section{Analyse:}

a. Le future et le conditionnel: -(e)r- : elle chantera...

Les morphes /er/,/r/ expriment les morphèmes grammaticaux \{future $\}$ et $\{$ conditionnel $\}$.

Exemples:

Indicatif futur : je danserai ; je viendrai ; j'aurai ; je serai

Conditionnel: je danserais ; je viendrais ; j'aurais ; je serais

\section{Extrait v:}

b. L'imparfait: -ai- pour les trois personnes du singulier et pour la troisième personne du pluriel et -i- pour la première et la deuxième personne du pluriel (l'imparfait).

\section{Analyse:}

Le morphe /ai/ représente le morphème grammatical \{imparfait\} pour les trois personnes du singulier et pour la troisième personne du pluriel et, le morphe / i/, pour la première et la deuxième personne du pluriel. Exemples:

$\begin{array}{cl}\text { Danser } & \text { aller } \\ \text { Je dansais } & \text { j'allais } \\ \text { Tu dansais } & \text { tu allais }\end{array}$




$\begin{array}{ll}\text { Il /Elle dansait } & \text { il /Elle allait } \\ \text { Nous dansions } & \text { nous allions } \\ \text { Vous dansiez } & \text { vous alliez } \\ \text { Ils/ elles dansaient } & \text { ils/elles dansaient }\end{array}$

Extrait vi:

c. Le passé simple et l'imparfait du subjonctif, temps de l'écrit, comportent quatre sorte de voyelles thématiques selon les verbes -a-, -i-, in-, et $-u-$.

Analyse:

Selon les verbes, les quatre morphes /a/, /i/, /in/, et /u/ expriment respectivement les morphèmes grammaticaux \{passé simple\} et \{imparfait du subjonctif\}.

Exemples:

Verbe (chanter): il chanta (passé simple);

qu'il chantât (imparfait du subjonctif)

Verbes (partir, prendre): il partit, il prit (passé simple);

qu'il partît ; qu'il prît (imparfait du subjonctif)

Verbe (venir): il vint (passé simple);

qu'il vînt (imparfait du subjonctif)

Verbe (recevoir): il reçut (passé simple);

$q u$ 'il reçût (imparfait du subjonctif)

3. 2. 2. Les morphes exprimant la personne et le nombre (Marques de personne et de nombre)

\section{Extrait vii:}

a. La première personne et la deuxième personne du pluriel sont toujours marquées respectivement par-ons et -ez, sauf dans (vous) faites, êtes, dites. Le passé simple comporte les formes particulières -mes, -tes: nous chantâmes, vous chantâtes.

Analyse:

Le morphe /ons/exprime le morphème grammatical $\left\{1^{\text {re }}\right.$ personne $\}$ $+\{$ pluriel $\}$, sauf au passé simple où ce morphème est réalisé par le morphe /mes/: Nous dansâmes.

Exemples:

Indicatif présent (danser, finir, vendre): nous dansons ; nous finissons; nous vendons

Future: nous danserons ; vous danserez ; nous finirons ; nous vendrons

Imparfait: nous dansions ; nous finissions ; nous vendions 
Conditionnel présent: nous danserions ; nous finirions ; nous vendrions

Subjonctif présent : que nous dansions; que nous finissions; que nous vendions

Subjonctif imparfait: que nous dansassions ; que nous finissions; que nous vendissions

Généralement, le morphe /ez/exprime le morphème grammatical $\left\{2^{\mathrm{e}}\right.$ personne $\}+\{$ pluriel $\}$.Mais /tes/ est le morphe particulier pour dire, êtes et faire conjugués (à l'indicatif présent) vous dites ; vous faites, et au passé simple pour tous les verbes (vous dansâtes/finîtes/ fûtes, etc.).

Exemples:

Indicatif présent: verbes (danser, finir, vendre):vous dansez; vous finissez; vous vendez

Future : vous danserez ; vous finirez; vous vendrez

Imparfait: vous dansiez, vous finissiez; vous vendiez

Conditionnel: vous danseriez; vous finiriez; vous vendriez

Subjonctif présent: que vous dansiez; que vous finissiez ;

que vous vendiez.

Subjonctif imparfait: que vous dansassiez; que vous finissiez; que vous vendissiez

\title{
Extrait viii
}

b. La troisième personne du pluriel se marque à l'écrit par $-\boldsymbol{t}$ (von -t), -nt (aime-nt) ou -ent (finiss -ent, chantai -ent). Le passe simple comporte une terminaison spécifique -rent: chantèrent, dirent.

Analyse:

Les morphèmes grammaticaux $\left\{3^{\mathrm{e}}\right.$ personne $\}+\{$ pluriel $\}$ se manifestent par les morphes / $\mathrm{t} /, \mathrm{nt} / \mathrm{ou} / \mathrm{ent} /$ :

\author{
Ils vont \\ Ils aiment \\ Ils finissent, dansaient
}

Mais au passé simple ils se réalisent par le morphe /rent/:

Elles dansèrent ; elles dirent...

\section{Extrait ix:}

c. À l'écrit, la deuxième personne du singulier est marquée par $-\mathbf{s}$ à tous les temps: tu chantes, tu finis, tu fais ( $-\boldsymbol{x}$ dans: tu veux, vaux, etc.), sauf à l'impératif présent des verbes du type chanter (chante).

Analyse:

À tous les temps, les morphèmes grammaticaux $\left\{2^{\mathrm{e}}\right.$ personne $\}+$ \{singulier\} sont représentés par le morphe $/ \mathrm{s} /$, mais ils se réalisent par $/ \mathrm{x} /$ 
dans vouloir (tu veux), valoir (vaux), pouvoir (peux), etc.). Voir par exemples, danser, finir, faire conjugués ci-dessous:

Présent : tu danses; tu finis; tu fais

Future: tu danseras; tu finiras ;tu feras

Imparfait: tu dansais ; tu finissais ; tu faisais

Passé simple: tu dansas; tu finis ; tu fais

Conditionnel: tu danserais ; tu finirais ;tuferais

Subjonctif présent: que tu danses ; que tu finisses; que tu fasses

Subjonctif imparfait: que tu dansasses; que tu finisses ; tu fisses

Mais: Absent dans le mode impératif: danse

\section{Extrait x:}

d. Selon le type de verbe, la troisième personne $d u$ singulier $d u$ présent de l'indicatif se marque à l'écrit par la consonne -t (finit, croit) ou se caractérise par une absence de marque (a, va, vainc). Aux temps autres que le présent de l'indicatif, la troisième personne est marquée par un -t final (chantait) ou ne possède pas de marque (chanta, chantera).

\section{Analyse:}

Selon le type de verbe, le morphe exprimant les morphèmes grammaticaux $\left\{3^{\mathrm{e}}\right.$ personne $\}+\{$ singulier $\}$ est tantôt $/ \mathrm{t} /$, tantôt zéro, comme le montrent les exemples cités ci- dessous:

$$
\begin{gathered}
\text { Il finit ; elle croit ;il dansait (verbes, au présent et à } \\
\text { l'imparfait) }
\end{gathered}
$$

\section{Quelques rappels:}

Les affixes flexionnels verbaux sont des signes àdouble face: le morphème et le morphe. Dans les modes impersonnels, nous avons les morphes exprimant les valeurs : l'infinitif et le présent, le participe et le présent, le gérondif, et le participe et le passé. Les modes personnels comportent les morphes correspondant aux morphèmes temporels et les morphes exprimant les morphèmes de personne et de nombre.Lesmorphèmes grammaticaux temporels sont représentés par des morphes (désinences, terme traditionnel) qui s'ajoutent immédiatement à morphème lexical (radical) et sont suivis des morphes réalisant les morphèmes (grammaticaux) de personne et de nombre. Tous les morphes sont syncrétiques(Charaudeau, 1992 ; Wouden, 1918), c'est-à-dire qu'un seul et même morphe exprime différents morphèmes grammaticaux (ou classes conceptuelles). Par exemple, le morphe / er/ dans manger permetd'exprimer à la fois deux morphèmes: les morphèmes \{infinitif\} et \{présent\}. En outre, le morphe /ons/ dans (nous) dansonspermet 
d'exprimer à la fois quatre morphèmes: les morphèmes \{indicatif\}, \{présent\}, $\left\{2^{\mathrm{e}}\right.$ personne\} et \{pluriel\}.

\section{Conclusion}

Dans ce travail, nous avons tenté de montrer que l'analyse en morphèmes semble être négligée dans le domaine de l'analyse des éléments de la langue française par les auteurs des ouvrages consacrés à la grammaire française ainsi que par les enseignants de la grammaire française dans les universités nigérianes. Nous avons donc fait une analyse en morphèmes de la flexion verbale en français pour servir d'exemple de l'analyse en morphèmes.Pour nous, aucun aspect de l'analyse des éléments du langage ne doit pas être abandonné, car on se rend pleinement à cette pensée de Defodon (1911): "Il faut remarquer qu'à tous ses degrés l'analyse emporte non seulement l'idée de division et de décomposition, mais aussi celle de recherche et d'invention. Elle est en effet plutôt une méthode de recherche et de découverte". Par conséquent, nous voudrions conseiller que l'étude de l'analyse en morphèmes doit être traitéedans les ouvrages consacrés à la grammaire française. En plus, nous conseillerions que les enseignants de la grammaire française dans les universités nigérianes font apprendre à leurs apprenants cet aspect de la morphologie pour que ceux-ci puissent jouir de son avantage:l'encouragement du développement intellectuel. Ceci est l'avis de Grosselin cité par Defodon (1911) lorsqu'il dit: "L'analyse doit être considérée avant tout comme une précieuse gymnastique intellectuelle". Cet article est considéré comme contribution à la production de documents pédagogiques destinés à l'enseignement et à l'apprentissage de l'analyse morphématique en français chez les apprenants du français langue étrangère, surtout ceux dans les universités nigérianes.

\section{References:}

1. Bako, G. C. (2010).La Grammaire fondamentale du français à l'usage des parents, des enseignants, des élèves et étudiants africains. Yaoundé: Edition CLÉ.

2. Bentolila, A. (2001). Grammaire : La référence pour tous avec les réponses immédiates à 700 questions d'usage. Paris: Nathan, Coll. « Les Guides Le Robert et Nathan ».

3. Bescherelle (2006).La grammaire pour tous: toutes les règles, des exemples commentés, des outils d'analyse, un index des difficultés. Paris: Hatier.

4. Charaudeau, P. (1992). Grammaire du sens et de l'expression. Paris: Hachette Livre.

5. Defodon, C. (1911). Analyse. Buisson, F. (Ed.), Nouveau dictionnaire de pédagogie et d'instruction primaire - [Ressource 
électronique].Paris: Librairie Hachette et Cie. http://www.inrp.fr/edition-electronique/lodel/dictionnaire-ferdinandbuisson/document.php?id=2042.

6. Dubois, J., Giacomo, M., Guespin, L., Marcellesi, C., Marcellesi, J.-B., \& Mével, J.-P. (2002). Dictionnaire de linguistique. Paris: Larousse.

7. Dubois, J., \& Lagane, R. (2004). La nouvelle grammaire du français. Paris: Larousse.

8. Ezeani, E. O. (2002). Éléments de langue française. Nimo: Rex Charles \& Patrick Ltd.

9. Gaillard, B., \& Colignon, J. (2005). Toute la grammaire.Paris: Magnard et Dicos d'or.

10. IPAM (2015).Grammaire du français $6^{\mathrm{e}} / 5^{\mathrm{e}}$. Edition no 33.Vanves: Edicef.

11. Martinet, A. (1970). Eléments de linguistique générale. $2^{\mathrm{e}}$ éd. Paris: Armand Colin.

12. Moeschler, J., \& Auchlin, A. (2005). Introduction à la linguistique contemporaine. Paris: Armand Colin.

13. Nekpo, C. F. (2013). Comment parlons-nous français? Etude syntaxique commentée de quelques mots et expressions courants. Deuxième Edition. Porto-Novo: Centre National de production de manuels scolaires.

14. Riegel, M., Pellat, J.-C., \& Rioul, R. (2014). Grammaire méthodique du français. $5^{\mathrm{e}}$ édition. Paris: Presse universitaire de France.

15. Rohr, A. (s.d.). «La morphologie». http://coursval.free.fr/coursL2/Morphologie(95).pdf.

16. Sanni, M. A. (2010). A Handbook on "Analyse grammatical".Badagry: Success Printers.

17. Touratier, C. (2002). Morphologie et morphématique: Analyse en morphèmes. Aix-en-Provence: Presses universitaires de Provence, https://books.openedition.org/pup/480

18. Wouden, T. "Dutch Morphology".http://www.taalportaal.org/taalportaal/topic/pid/topic14244200627059845. 